\title{
A Study Proposal of Individualized Educational Program Development Strategies Based on Single Subject Studies
}

\author{
Le Qi ${ }^{*}$ and $\mathrm{Bo} \mathrm{Su}$
}

\author{
Aviation Maintenance NCO Academy, Air Force Engineering University \\ *Corresponding author.Email: qixiaole@buaa.edu.cn
}

\begin{abstract}
The problem of underachieved students in vocational education is a painful point in improving the quality of higher vocational education. By introducing a small sample and non-statistical data research method of single subject research into the process of teaching reform in our school, we are able to use the natural science method of behavioral science to guide the practice of teaching reform through the construction of a detailed observation system and data analysis system. This paper presents the ideas, methods, steps, and technical approaches for the implementation of this study proposal, and provides a practical reference for practitioners of teaching reform.
\end{abstract}

Keywords: Higher vocational education, Teaching reform, Single subject study, Observation system, Data analysis

\section{INTRODUCTION}

Vocational education is a very important branch of higher education, and non-commissioned officer vocational education is a special branch of vocational education, which has both the same characteristics of general vocational education and the special characteristics of military vocational education. Only strict standards can ensure that every student who passes the final examination has excellent military skills and professional quality.

The last-place elimination system is a way to ensure the quality of talent, but it is by no means the best method. Under these circumstances, it is necessary to be responsible as an instructor and to use all available resources to improve the overall compliance rate of all cadets. Due to the unique nature of military academies and the need for uniformity, the frequency of free access to learning materials is influenced by factors such as confidentiality; therefore, it is necessary to consider the overall situation and rationally combine existing educational resources to build and allocate, and design individualized educational programs for underachieved students.

\section{LITERATURE REVIEW}

Effective use of instructional and assessment data is needed to make scientifically based decisions about individualized educational programs. Statistical analysis is commonly used in the analysis of instructional data. Statistical procedures allow the researcher to objectively determine whether the observed effects are accidental or the inevitable result of the treatment.

Non-statistical, small-sample research methods have also been developed in both basic and applied research. Among them, the single-subjects research method is an empirical design and data analysis method for small samples that provides rapid feedback on the effectiveness of an instructional intervention and is suitable for the development and implementation of individualized instructional programs for individuals who are behind.

Single-subject studies are rarely used in China, and the theory is relatively understudied. Single subject studies are appropriate for studies with small sample sizes and high heterogeneity between samples. Special education is individualized, which is consistent with single subject studies. For this reason, single subject studies are popular among researchers in the field of 
special education, especially abroad, such as Hammond and Gast (2010) who reviewed various single subject study designs published in eight relevant journals between 1983 and 2007. The authors reviewed one question per volume per year, for a total of 1936 articles published in 196 journals, 456 of which were published using a single subject study design [4]. The singlesubject research approach is very popular in the field of special education in foreign countries. However, when I searched for articles on the Internet using "single subject study" as a keyword, I could only collect nine articles.

\section{SIGNIFICANCE OF THE STUDY}

The classroom revolution underway at our school usually focuses more on improving the learning outcomes of the average student, but statistics from past classes show that there is always one or a few underachievers in each class, who work hard enough, but their results are always between a pass and a fail. However, this group of students does not end up in jobs that are different from the best of the students, and their abilities are not diminished for their own reasons; therefore, faculty members must take responsibility for developing targeted, individualized educational programs for this group of students. We will conduct research on educational intervention measures so that the classroom revolution and educational reform measures will also benefit this niche group, ultimately achieving the goal of improving the overall learning effect and providing excellent and qualified personnel to serve the job needs of students.

Statistical data show that these students have difficulty benefiting from general education instructional supports and need more powerful tools and interventions to catch up during their short time in school. The introduction of a small, non-statistical data analysis tool, such as a single-subject study, would allow instructors to quickly adjust educational strategies, dynamically adjust and deploy teaching resources and tools to improve teaching effectiveness based on the results of the data, and provide options to address this common pain point in vocational higher education.

\section{RESEARCH METHODOLOGY}

By using natural science research ideas and introducing a single-subject experimental design, changes in instructional strategies and program changes are based on objective data analysis to reduce arbitrariness in decision making and increase the probability of success of the intervention. That is, we collect data on the teaching and learning process, analyze the baseline logic of the effect and the trend of the frequency of typical target behaviors after the intervention to test the effectiveness of existing strategies, and guide the next practice of changing teaching and learning strategies to achieve the desired results.

\subsection{Research Goals}

Using scientific experimental design and data analysis tools, find an evidence-based, easy-toimplement paradigm for personalized educational programs for underachieved students.

\subsection{Research Content}

1. Define academic underachievers, determine assessment methods and criteria.

Example definition of academic underachievers:

Students that are behind in three past major subjects in academic achievement.

Example assessment criteria:

Above average scores of formative assessments are steadily observed and confirmed in three subjects.

Note that the criteria used here is not a final score of one subject but many continuous formative assessments. We are convinced that these are true indicators that can predict further academic successes.

Assessment method:

Whatever means used in traditional classrooms can be used in the scheme as long as objectiveness can be assured during the assessment.

2. Establishing observing systems, defining observing indicators, and training observing personnel.

Sample observing system:

Underachieved students must be carefully monitored such that their performance can be observed and recorded with high confidence.

\section{Parameters chosen:}

Since the assessment criteria has been set to be above average scores of various formative assessments, parameters to be monitored and observed should be in accordance with the above criteria, e.g., a practice class should use fluency as an indicator to show the competitiveness of the student under study. Rate of response is an indicator that is associated with every human operant behavior and is therefore a basic parameter we may use to observe and record during this study proposal.

Training of observers:

In most cases, classroom observations do not require extra skills or equipment. However, a teacher may be quite occupied during the class time when recorders might come in handy such that fidelity of observations 
can be guaranteed. Inter-observer agreement is another factor that a recorder is usually a crosscheck necessity.

3. Establish baseline logic and specific scenarios experimental design.

\section{Baseline:}

The raw data obtained from the act of measuring in the absence of variables by adding them to the holding pattern is called the baseline. From a purely scientific or analytical point of view, the primary purpose of establishing a baseline is to use the subject's performance in the absence of the variant as an objective basis for detecting the effects of future presentations of the variant.

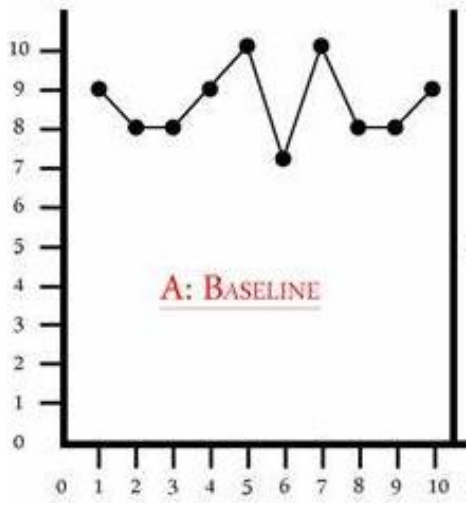

Figure 1 Example baseline

During baseline establishment, it is commonly agreed that at least five points should be guaranteed to ensure a steady trend judgement. For example, if we are to collect the formative assessment baseline achievement for a subject, five observations must be carried out minimum.

Typical scenarios experimental design:

\section{Single Subject Designs} A-B-A-B (reversal)

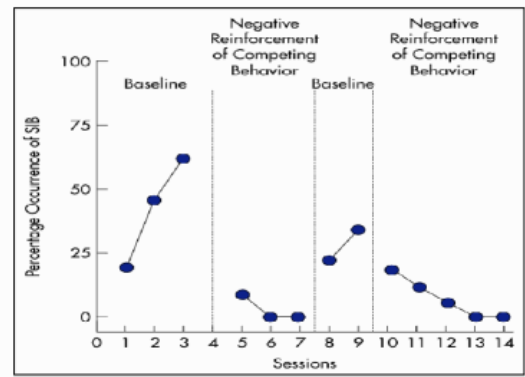

Figure $2 \mathrm{ABAB}$ experimental design

Typical scenarios are usually closely connected with our goal of educational intervention. Suppose we want to improve the performance of Jackie's in subject A, then subject $\mathrm{A}$ class must be a typical scenario in our study.An experimental design that includes at least one subject, a baseline period, an intervention period, an independent variable, and a dependent variable to answer the question to be studied or to test a hypothesis.

4. Data collection and visualization.

Data collection:

Dropping a dot on a data collection sheet is a first step toward data driven decision making. The actual process is not different with traditional class implementation. However, an observing plan must be made and strictly carried out as planned such that we can have concrete data.

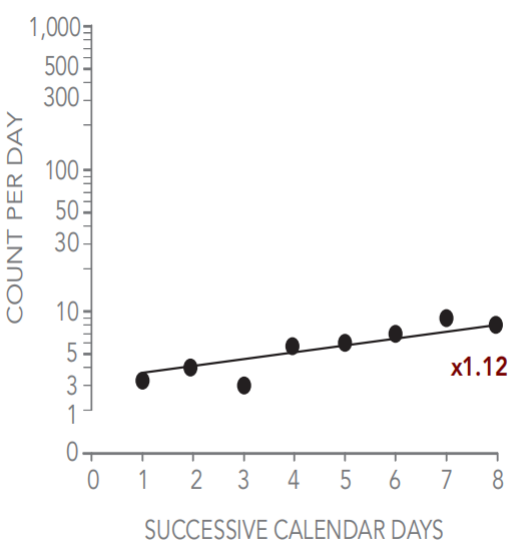

Figure 3 Data Visualization example [8]

Data visualization:

Data visualization has no unique way. Whatever comes in handy can be applied. Normally, as can be seen from the above figure, visual display generated from Excel can meet most of the needs while more advanced tools like Tableau can also be used.

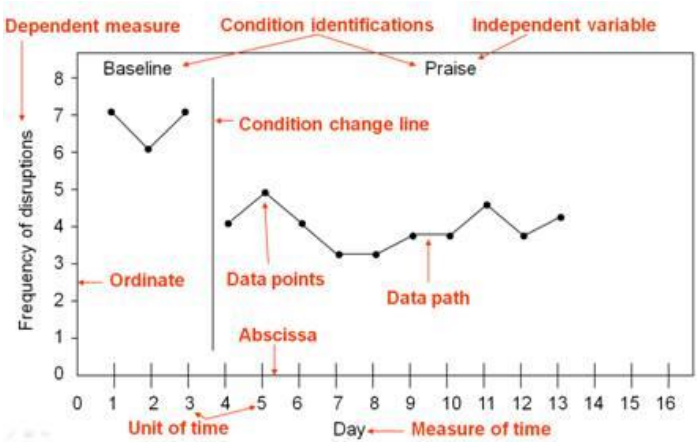

Figure 4 Data visualization example

5. Interpreting data and adjusting instructional strategies based on results.

Data analysis:

Analyze the stability and trends of the data generated by the trial to determine the effectiveness of the instructional intervention strategy and, accordingly, 
to continue or discontinue the current instructional strategy.

6. Dynamic tracking until standards are met.

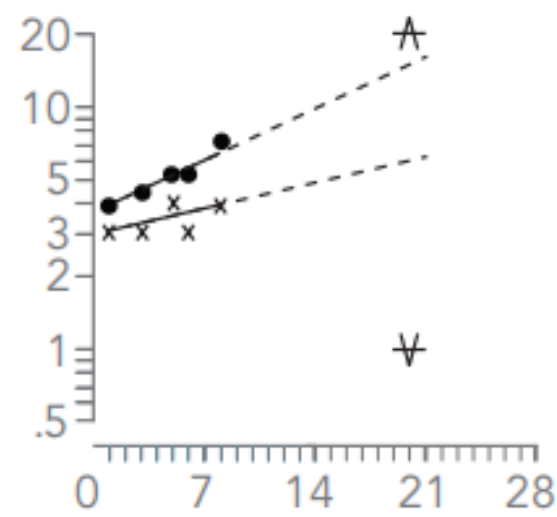

Figure 5 Decision making using standard celeration chart [8]

The projection lines (dashed lines) tell that the subject under study can not meet his originally set goal and thus adjustment of measures needs to be taken accordingly. Keep on collecting data and displaying them visually, stick to the promising plan based on better performance and faster progressing speed, study goals will be achieved along on an optimized learning trajectory.

\section{CASE DEMONSTRATION}

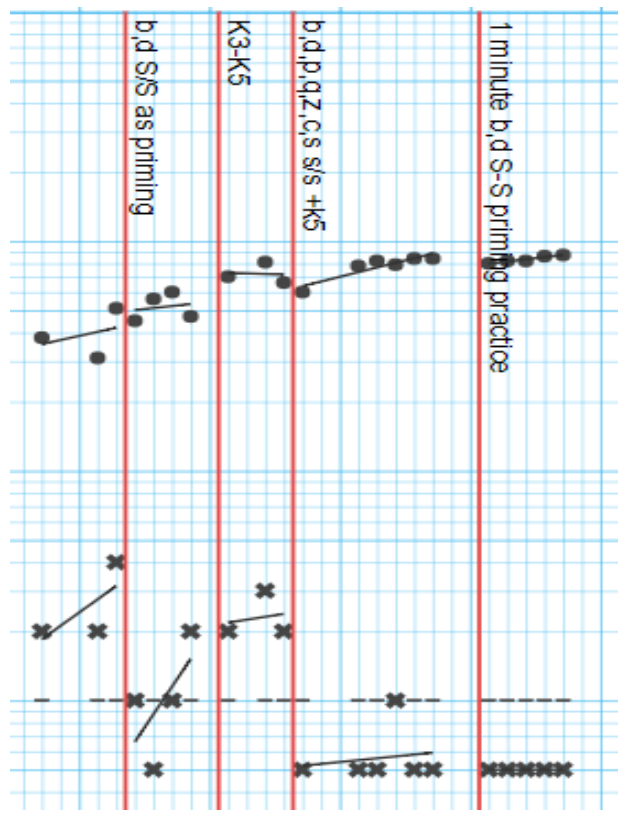

Figure 6 Demonstration of SSC

As is shown in Figure 6 the rate of response was used as an indicator of student Jackie's performance, the first red vertical line separates the baseline and the first stage of educational intervention while the other stages between other vertical lines. As can be seen from the chart, although Jackie's the mistakes fluctuated until the last stage of intervention was carried out, his performance had a steady improvement after the baseline period. After adjusting Jackie's IEP plans, study goals have been achieved within two months' time, which is an optimized outcome compared with traditional educational solutions for typical underachievers.

\section{CONCLUSION}

Small and medium samples of special education and non-statistical data research methods for single-subject research was introduced into the practice of teaching reform in vocational education in our school.

By applying the proposed frame, observation system and data analysis system, the process of education reform can be guided with visual tools using the natural science method of behavioral science, thus improving accuracy and efficiency. The proposed method can be used to guide the ongoing educational reform in our school and can also be referred to elsewhere if needed.

\section{AUTHORS' CONTRIBUTIONS}

Natural science method is introduced into education reform in an easy to operate way in details.

\section{ACKNOWLEDGMENTS}

Special thanks to my partner Bo Su for his support.

\section{REFERENCES}

[1] Virues-ortega J Single Subject Research Design $[\mathrm{M}] /$ The SAGE Encyclopedia of theory in Psychology 2016.

[2] Logan LR Single subject research design: Recommendations for levels of evidence and quality rating $[\mathrm{J}]$ Developmental Medicine \& Child, Neurology 2008.

[3] Campbell Using a single subject research method to evaluate the effectiveness of treatment $[\mathrm{J}]$ The American Journal of Occupational Therapy: Official publication of the American Occupational Therapy Association 1988.

[4] Hammond D \&Gast D L Descriptive analysis of singlesubject research [J].Education and Training in Autism and Developmental Disabilities, 2010(45).

[5] Xiaozhang Wen, Zesen Huang, Journal of Mianyang Normal College, ollege, ng Normal College, ezed Educational Planning in Higher Vocational EducationC, 2008. 
[6] Fei Ke. Journal of Suihua Normal College, lege, mal College, , e, nal EducationCollege, es, 2010(45).ate way in

[7] Wen Liu. Journal of Human Normal University. EducationCollege, es, 2010(45).Experiments in Education and Psychologysity. E
[8] Richard Kubina, ebook 2018, "Change behavior change lives precisely", 2018, pp26. 